PROCEEDINGS OF THE

AMERICAN MATHEMATICAL SOCIETY

Volume 131, Number 6, Pages 1717-1725

S 0002-9939(03)06909-0

Article electronically published on January 15, 2003

\title{
LEAFWISE HOLOMORPHIC FUNCTIONS
}

\author{
R. FERES AND A. ZEGHIB
}

(Communicated by Michael Handel)

\begin{abstract}
It is a well-known and elementary fact that a holomorphic function on a compact complex manifold is necessarily constant. The purpose of the present article is to investigate whether, or to what extent, a similar property holds in the setting of holomorphically foliated spaces.
\end{abstract}

\section{Introduction AND STATEMENT OF RESULts}

Suppose that $M$ is a compact manifold, $\mathcal{F}$ is a continuous foliation of $M$ by (not necessarily compact) complex leaves, and that $f$ is a continuous leafwise holomorphic function. The question we study is whether or not $f$ must be leafwise constant.

We will actually work in the setting of foliated spaces, as defined in [3]. Thus $M$ is only a topological space, while the leaves of $\mathcal{F}$ admit a smooth manifold structure that varies continuously on $M$. The term foliated manifold will be reserved for when $M$ has a differentiable structure relative to which each leaf of $\mathcal{F}$ is $C^{1}$ immersed and the foliation tangent bundle, $T \mathcal{F}$, is a $C^{0}$ sub-bundle of $T M$. In all situations, it will be assumed that $M$ is compact and connected. We also assume that $(M, \mathcal{F})$ is a holomorphically foliated space, by which we mean that each leaf of $\mathcal{F}$ carries the structure of a complex manifold and that this structure varies continuously on $M$. (The definition of foliated spaces given in [3] readily extends to the present case in which leaves are complex manifolds.) The foliated space $(M, \mathcal{F})$ may, on occasion, also carry a leafwise Hermitian metric (resp., is leafwise Kähler); that is, the leaves of $\mathcal{F}$ may carry a smooth Hermitian metric (resp., a Kähler metric) that, together with all its derivatives along leaves, varies continuously on $M$.

If the foliation is such that any continuous leafwise holomorphic function is leafwise constant, we will say that $(M, \mathcal{F})$, or simply $\mathcal{F}$, is holomorphically plain. It will be proved that a number of general classes of holomorphically foliated manifolds are holomorphically plain. We also give an example of a real analytic, non-plain, holomorphically foliated manifold (with real analytic leafwise holomorphic functions that are not leafwise constant) and indicate a general construction that shows how such examples can be obtained.

When the leaves of $\mathcal{F}$, individually, do not support non-constant bounded holomorphic functions, then clearly $\mathcal{F}$ is holomorphically plain. This is the case, for example, if the universal covering spaces of the leaves are isomorphic as complex

Received by the editors July 14, 2001.

2000 Mathematics Subject Classification. Primary 37C85; Secondary 32A99.

Key words and phrases. Foliated spaces, leafwise holomorphic functions. 
manifolds to $\mathbb{C}^{n}$. Therefore, the question only becomes meaningful for cases such as, say, a foliation by Riemann surfaces containing leaves that are isomorphic to the unit disc. Since individual leaves may now support non-constant bounded holomorphic functions, it becomes necessary to understand the constraining role played by the foliation dynamics.

The subject of this article has a natural counterpart for foliations with leafwise Riemannian metrics and functions that are leafwise harmonic. There is, as well, a discretized form of the problem (of deciding whether leafwise harmonic functions are leafwise constant) in the setting of actions of finitely generated groups and functions that are harmonic along orbits for a combinatorial Laplacian. These "harmonic" variants of the subject contain some essential additional difficulties and, for the sake of keeping this article as elementary as possible, they will be treated elsewhere.

The first author wishes to thank the members of the UMPA - ENS Lyon, and FIM - ETH Zürich for their hospitality while this work was being written.

1.1. Group actions and foliated bundles. Holomorphically foliated spaces arise in a number of ways. For example, as the orbit foliation of a locally free $C^{1}$ action of a complex connected Lie group on a compact manifold, or as a foliated bundle over a compact connected complex manifold. See also the work of E. Ghys [4] on laminations by Riemann surfaces and leafwise meromorphic functions.

It is a simple fact that if $(M, \mathcal{F})$ is the orbit foliation of a continuous locally free action of a connected complex Lie group $G$, then $\mathcal{F}$ is holomorphically plain, where the complex structure on leaves is the one that makes the orbit map $o_{x}: g \mapsto g x$ (from $G$ onto the leaf of $x$ ) a local isomorphism of complex manifolds. We have the following slightly more general fact.

Proposition 1.1. Let $(M, \mathcal{F})$ be a holomorphically foliated space such that $T \mathcal{F}$ is holomorphically trivial. Then $\mathcal{F}$ is holomorphically plain.

Proof. The hypothesis that $T \mathcal{F}$ is holomorphically trivial means the following: there exist vector fields, $X_{1}, \ldots, X_{l}$, on $M$, where $l$ is the leaf dimension, such that the $X_{i}$ are everywhere tangent to $\mathcal{F}$, linearly independent, and define holomorphic vector fields on leaves. Furthermore, the $X_{i}$, together with their tangential derivatives of first order, are continuous on $M$.

Since $M$ is compact, the $X_{i}$ are complete vector fields and each flow line is the image of a holomorphic map from $\mathbb{C}$ into a leaf. Therefore, the restriction to orbits of leafwise holomorphic functions define bounded holomorphic functions on $\mathbb{C}$. As a result, such functions are constant on orbits, hence leafwise constant.

The next corollary follows from the proposition and a simple remark due to Wang.

Corollary 1.2. If $(M, \mathcal{F})$ is a holomorphically foliated space having a dense leaf and $T \mathcal{F}$ is holomorphically trivial, then $\mathcal{F}$ is the orbit foliation of a locally free action of a connected complex Lie group.

Proof. Write $\left[X_{i}, X_{j}\right]=\sum_{k} f_{i j}^{k} X_{k}$. The coefficients $f_{i j}^{k}$ are continuous, leafwise holomorphic, hence leafwise constant. Due to the existence of a dense leaf, these coefficients are constant on $M$. Therefore, the $X_{i}$ span a finite-dimensional Lie algebra. The corollary is now a result of standard facts in Lie theory. 
A much more interesting class of examples consists of foliated bundles. We now recall the definition of foliated bundles in the special setting that concerns us here.

Let $S$ denote a compact connected complex manifold, let $\tilde{S}$ be the universal covering space of $S$, and denote by $\Gamma$ the group of deck transformations of $\tilde{S}$. The action of $\gamma \in \Gamma$ on $p \in \tilde{S}$ will be written $p \gamma$. Let $X$ be a compact connected space on which $\Gamma$ acts by homeomorphisms. The latter action can be represented by a homomorphism $\rho: \Gamma \rightarrow \operatorname{Homeo}(X)$ from $\Gamma$ into the group of homeomorphisms of $X$. We write simply $\gamma(x)=\rho(\gamma)(x)$, but we use the symbol $\rho$ to indicate which action on $X$ is being used.

Then $\Gamma$ acts on the product $\tilde{S} \times X:(p, x) \gamma:=\left(p \gamma, \gamma^{-1}(x)\right)$, for $p \in \tilde{S}, x \in$ $X$ and $\gamma \in \Gamma$. Let $M:=(\tilde{S} \times X) / \Gamma$ be the space of $\Gamma$-orbits. The natural projection $\pi: M \rightarrow S$ gives $M$ the structure of a fiber bundle over $S$ whose fibers are homeomorphic to $X$, and $M$ is foliated by complex manifolds, transversal to the fibers of $\pi$, which are coverings of $S$. The resulting foliated space will be written $\left(M_{\rho}, \mathcal{F}_{\rho}\right)$.

Note that the dynamics of a foliated bundle is largely determined by the dynamics of the $\Gamma$-action on $X$. Thus, for example, if $X$ admits a $\Gamma$-invariant finite measure of full support (respectively, if the $\Gamma$-action has a unique minimal set), then $M_{\rho}$ admits a completely invariant finite measure of full support (respectively, $\left(M_{\rho}, \mathcal{F}_{\rho}\right)$ has a unique minimal set). It should also be clear that $x \in X$ has a finite $\Gamma$-orbit if and only if $\tilde{S} \times\{x\}$ maps to a closed leaf of $\mathcal{F}_{\rho}$.

1.2. Leafwise Hermitian and Kähler foliations. The results in this section assume that $(M, \mathcal{F})$ is provided with a (continuous on $M$ ) leafwise Hermitian metric $\langle\cdot, \cdot\rangle$. We denote by $\Omega$ the associated leafwise volume form and define the divergence of a continuous leafwise smooth vector field $X$ to be the function $\operatorname{div} X$ such that $\mathcal{L}_{X} \Omega=(\operatorname{div} X) \Omega$, where $\mathcal{L}_{X}$ denotes the Lie derivative along $X$. A Borel measure $m$ on $M$ is said to be completely invariant if

$$
m(\operatorname{div} X):=\int_{M} \operatorname{div} X(x) d m(x)=0
$$

for every continuous, leafwise smooth $X$. Completely invariant measures are equivalent to holonomy invariant transverse measures on $(M, \mathcal{F})(\mathrm{cf}$. [5]).

A measure $m$ on $M$ is said to have full support if its support coincides with $M$.

Proposition 1.3. If $(M, \mathcal{F})$ is a leafwise Kähler foliated space that admits a completely invariant measure of full support, then $\mathcal{F}$ is holomorphically plain.

Let $\bar{\partial}^{*}$ denote the adjoint operator to $\bar{\partial}$ with respect to the chosen leafwise Hermitian metric, and define the $\bar{\partial}$-Laplacian on leafwise smooth functions by $\Delta_{\bar{\partial}}=$ $\bar{\partial}^{*} \bar{\partial}$ (we use the notation and sign conventions of [11]). A Borel measure $m$ on $M$ will be called $\Delta_{\bar{\partial}}$-harmonic, or simply harmonic, if

$$
m\left(\Delta_{\bar{\partial}} h\right):=\int_{M}\left(\Delta_{\bar{\partial}} h\right)(x) d m(x)=0
$$

for all continuous, leafwise smooth functions $h: M \rightarrow \mathbb{C}$.

Since, on a Kähler manifold, $\Delta_{\bar{\partial}} h=-\frac{1}{2} \operatorname{div} \operatorname{grad} h$, Proposition 1.3 is an immediate consequence of the following.

Proposition 1.4. Suppose that $(M, \mathcal{F})$ is a leafwise Hermitian foliated space. Also suppose that the union of the supports of all $\Delta_{\bar{\partial}}$-harmonic measures is $M$. Then $(M, \mathcal{F})$ is holomorphically plain. 
A leafwise holomorphic function $f$ is $\Delta_{\bar{\partial}}$-harmonic since $\bar{\partial} f=0$, so Proposition 1.4 is a corollary of [6, Theorem $1(\mathrm{~b})]$. When $(M, \mathcal{F})$ is leafwise Kähler, a much simpler, independent proof is obtained by observing the following. It is well known (see, for example, [8, p. 115]) that under this extra hypothesis $\Delta_{\bar{\partial}} \bar{f}=\Delta_{\partial} \bar{f}=0$, so we also have $\Delta_{\bar{\partial}} \bar{f}=0$. Another elementary calculation gives the identity

$$
\Delta_{\bar{\partial}}\left(|f|^{2}\right)=-\|\partial f\|^{2}+f \Delta_{\bar{\partial}} \bar{f}=-\|\partial f\|^{2} .
$$

By integrating the terms in the equality against a harmonic measure $m$ one deduces that $\partial f=0 m$-almost everywhere, and as $\partial f$ is continuous, it is zero on the support of $m$. Therefore, $f$ must be constant on that support.

1.3. Foliations with few minimal sets. Unless specified otherwise, $(M, \mathcal{F})$ will continue to denote a compact, connected, holomorphically foliated space. We recall that a minimal set $X$ of $(M, \mathcal{F})$ is a closed, nonempty, $\mathcal{F}$-saturated subset of $M$ that has no proper subset with these same properties. If $M$ is, itself, a minimal set, then $\mathcal{F}$ is said to be a minimal foliation.

Proposition 1.5. Suppose that the closure of each leaf of $(M, \mathcal{F})$ contains (at most) countably many minimal sets. Then $\mathcal{F}$ is holomorphically plain.

The next lemma and corollary will be needed a number of times in the rest of the paper.

Lemma 1.6. Let $(M, \mathcal{F})$ be a holomorphically foliated compact connected space and let $f: M \rightarrow \mathbb{C}$ be a leafwise holomorphic function. Let $C \subset M$ be the set where $f$ is leafwise constant. Suppose that $f(C) \subset \mathbb{C}$ is at most countable. Then $f$ is constant on $M$.

Proof. Clearly $C$ is a compact $\mathcal{F}$-saturated set. By the open mapping theorem for holomorphic functions, the restriction of $f$ to each leaf in the complement of $C$ is open, hence $f$ itself is an open mapping on that complement. Therefore $U:=f(M \backslash C)$ is a bounded open subset of $\mathbb{C}$. Since $M$ is compact, $f(M)=U \cup f(C)$ is compact. In particular, the boundary of $U$ is contained in $f(C)$, which is by assumption a countable set. But a bounded nonempty open set in $\mathbb{C}$ cannot have a countable boundary. Therefore $U$ is empty and (as $M$ is connected) $f(M)$ reduces to a point.

Corollary 1.7. Suppose that $(M, \mathcal{F})$ has (at most) countably many minimal sets. Then any leafwise holomorphic function is constant on $M$.

Proof. Let $f$ be a leafwise holomorphic function and let $C$ be as in Lemma 1.6] Denote by $\mathcal{M}$ the union of minimal sets. We claim that $f(C)=f(\mathcal{M})$. Indeed, if $x \in C$ and $f(x)=c$, then $f$ takes the constant value $c$ on the closure of the leaf containing $x$. But this closure contains a minimal set, so $f(C) \subset f(\mathcal{M})$. Conversely, by an application of the maximal principle for holomorphic functions, each closed saturated set contains leaves where $f$ is constant, so $f(C)=f(\mathcal{M})$ as claimed. The main assertion is now a consequence of Lemma 1.6.

By applying Corollary 1.7 to the closure of each leaf we obtain Proposition 1.5. Proposition [1.5 clearly applies to minimal foliations. Also note that if $\mathcal{F}$ is a (transversely) Riemannian foliation, then the closure of each leaf is a minimal set [13], so the proposition also applies. Therefore we get the next corollary, which will 
be used later a number of times. (Note that a compact group of diffeomorphisms of a compact manifold must preserve a Riemannian metric.)

Corollary 1.8. Let $\left(M_{\rho}, \mathcal{F}_{\rho}\right)$ be a holomorphically foliated bundle over $S$ with fiber a compact manifold $X$, where the homomorphism $\rho$ has values in a compact group of diffeomorphisms of $X$. Then $\mathcal{F}_{\rho}$ is holomorphically plain.

There is also a large class of foliated bundles associated to $\Gamma$-actions on projective space $\mathbb{F} P^{n}, \mathbb{F}=\mathbb{R}$ or $\mathbb{C}$, and derived from linear representations of $\Gamma$, for which the hypothesis of the proposition are satisfied. This will be described after introducing some notation. Let $G L(W)$ be the group of linear automorphisms of $W$, where $W$ is a vector space over $\mathbb{F}$. The quotient of $G L(W)$ by its center will be written $P G L(W)$ and the projective space associated to $W$ will be written $P(W)$. If $W=\mathbb{F}^{n}$, we write $P G L(W)=P G L(n, \mathbb{F})$ and $P(W)=\mathbb{F} P^{n-1}$.

Let $\rho: \Gamma \rightarrow G L(W)$ be a linear representation of a group $\Gamma$ on a vector space $W$. An element $\gamma \in \Gamma$ will be called proximal if the maximal characteristic exponent of $\rho(\gamma)$ is simple. The next result is a consequence of Proposition[1.12] and [12] 3.4 and 3.6, Chapter VI].

Proposition 1.9. Let $S$ be a connected, compact, complex manifold with fundamental group $\Gamma, W$ an n-dimensional vector space over $\mathbb{F}$, and $\rho: \Gamma \rightarrow G L(W)$ a continuous homomorphism for which $\Gamma$ contains a proximal element. Let $\left(M_{\rho}, \mathcal{F}_{\rho}\right)$ be the foliated bundle over $S$ with fiber $P(W)$, where $\Gamma$ acts on $P(W)$ via $\rho$. Then $\left(M_{\rho}, \mathcal{F}_{\rho}\right)$ is holomorphically plain.

The hypothesis of Proposition 1.9 holds if the image of $\Gamma$ in $P G L(W)$ is Zariski dense and not precompact [12, Theorem 4.3(i)]. If the image is precompact, we can apply Corollary [1.8, so the following corollary holds.

Corollary 1.10. Let $S$ be a connected, compact, complex manifold with fundamental group $\Gamma, W$ an n-dimensional vector space over $\mathbb{F}$, and $\rho: \Gamma \rightarrow G L(W)$ a continuous homomorphism such that the image of $\Gamma$ in $P G L(W)$ is Zariski dense. Let $\left(M_{\rho}, \mathcal{F}_{\rho}\right)$ be the foliated bundle over $S$ with fiber $P(W)$, where $\Gamma$ acts on $P(W)$ via $\rho$. Then $\left(M_{\rho}, \mathcal{F}_{\rho}\right)$ is holomorphically plain.

It will follow from Corollary 1.10 that foliated bundles associated to projective (linear) actions of $\pi_{1}(S)$, for a compact Riemann surface $S$, are generically holomorphically plain, in the sense described below.

We first recall some definitions. Let $S=\mathbb{D} / \Gamma$ be a surface of genus $g \geq 2$, where $\mathbb{D}$ denotes the Poincaré disc and $\Gamma$ is a cocompact discrete group (without torsion) of hyperbolic isometries. Let $G$ be an algebraic group and denote by $\operatorname{Hom}(\Gamma, G)$ the variety of homomorphisms from $\Gamma$ to $G$.

Theorem 1.11. Let $G=G L(n, \mathbb{C})$. Then there is a Zariski open dense subset $U$ in $\operatorname{Hom}(\Gamma, G)$ such that, for each $\rho \in U$, the foliated bundle $\left(M_{\rho}, \mathcal{F}_{\rho}\right)$ for the corresponding $\Gamma$-action on $\mathbb{C} P^{n-1}$ is holomorphically plain.

Proof. By [14], $\operatorname{Hom}(\Gamma, G)$ is irreducible, and by [1, 8.2] the homomorphisms with Zariski dense image form a Zariski open subset $\mathcal{D} \subset \operatorname{Hom}(\Gamma, G)$. On the other hand, $\mathcal{D}$ is clearly nonempty. ( $\Gamma$ has homomorphisms onto the free group on 2 generators and it is easy to show that the free group has representations with Zariski dense image.) Therefore, the theorem is a consequence of Corollary 1.10 
A topological space $X$ equipped with an action of a group $\Gamma$ by homeomorphisms will be called here a $\Gamma$-space of convergence type if the following holds: there exists a countable (possibly finite) family of subsets $X_{i} \subset X, i=1,2, \ldots$, such that (i) the intersection of all the $X_{i}$ is countable and (ii) for each $i$ there is a sequence $\gamma_{m} \in \Gamma$ and a point $x_{i} \in X$ such that $\gamma_{m}(y)$ converges to $x_{i}$ as $m \rightarrow \infty$, for each $y$ in the complement of $X_{i}$.

As a simple example, let $X=\mathbb{C} P^{1}$ and let $\rho: \Gamma \rightarrow P S L(2, \mathbb{C})$ be a homomorphism such that $\rho(\Gamma)$ is not relatively compact. Then $X$, with the $\Gamma$-action obtained from $\rho$, is a $\Gamma$-space of convergence type. The $\Gamma$-actions on $\mathbb{F} P^{n-1}$ of Proposition 1.9 as well as the natural action of any infinite subgroup $\Gamma$ of a Gromov-hyperbolic group $G$ on the boundary $\partial G$, also define convergence $\Gamma$-spaces.

Proposition 1.12. Let $S$ be a compact complex manifold with fundamental group $\Gamma$, let $X$ be a compact $\Gamma$-space of convergence type, and let $(M, \mathcal{F})$ be the corresponding foliated bundle over $S$. Then $\mathcal{F}$ is holomorphically plain.

Proof. We identify $X$ with a fixed fiber of the foliated bundle, so that the holonomy transformations of $\mathcal{F}$ correspond to the $\Gamma$-action on $X$.

Suppose that $f$ is a leafwise holomorphic continuous function on $M$. Let $C \subset X$ be the compact $\Gamma$-invariant subset that corresponds to leaves on which $f$ is constant. Then there is a countable set of points $x_{1}, x_{2}, \ldots$ such that for all $y \in C$, with the possible exception of a countable subset of $C$, we can find a sequence $\gamma_{m}$ such that $\gamma_{m} y \rightarrow x_{i}$ for some $i$. Clearly the $x_{i}$ belong to $C$. Therefore $f$ can take at most a countable set of values on leaves of $\mathcal{F}$ in $C$. So $f$ must be leafwise constant by Lemma 1.6

A class of $\Gamma$-spaces for which the convergence property is well known to hold consists of actions of nonrelatively compact subgroups of a Gromov-hyperbolic group on the boundary of the latter. (See, for example, 7] and references cited there. It should be noted that the standard definition of the convergence property used in the literature on hyperbolic groups is much more restrictive than the one we are using here.) Therefore the following holds.

Proposition 1.13. Let $G$ be a Gromov-hyperbolic group, $X$ the boundary of $G$, and $S$ a compact connected complex manifold with fundamental group $\Gamma$. Suppose that $\Gamma$ acts on $X$ via a homomorphism $\rho: \Gamma \rightarrow G$ and let $\left(M_{\rho}, \mathcal{F}_{\rho}\right)$ be the corresponding foliated bundle over $S$. Then $\mathcal{F}_{\rho}$ is holomorphically plain.

Corollary 1.14. Let $S$ be a compact Riemann surface, let $\rho: \Gamma \rightarrow G$ be a homomorphism of the fundamental group of $S$ into a connected simple Lie group of rank one, and let $\left(M_{\rho}, \mathcal{F}_{\rho}\right)$ be the foliated bundle over $S$ with fibers $X$, where $X$ is the boundary at infinity of the Riemannian symmetric space associated to $G$. Then $\mathcal{F}_{\rho}$ is holomorphically plain.

1.4. Codimension-one. The main idea used in the proof of Proposition 1.5 together with elementary facts about the structure of codimension-one foliations yield the following.

Theorem 1.15. If $(M, \mathcal{F})$ has codimension 1 , then it is holomorphically plain.

Proof. The basic facts about codimension-one foliations that we use can be found in [10] or [3], for example. We recall that a minimal set is said to be exceptional if it is neither a single closed leaf of (a codimension-one foliation) $\mathcal{F}$ nor all of $M$. 
By a theorem of Haefliger [9] (see also [10]), the union of compact leaves of $\mathcal{F}$ is a compact set, which we denote by $N$. Let $L$ be a connected component of the complement of $N$. Then, as $\mathcal{F}$ has codimension 1 , the closure $\bar{L}$ is a compact manifold whose boundary is a finite union of compact leaves (cf. [10]).

It is known that $(M, \mathcal{F})$ has only a finite set of exceptional minimal sets. In particular, $\left(\bar{L},\left.\mathcal{F}\right|_{\bar{L}}\right)$ has finitely many minimal sets (exceptional or not). Applying Corollary 1.7 we deduce that any leafwise holomorphic function is constant on $\bar{L}$. In particular, any leafwise holomorphic function is leafwise constant on $M \backslash N$. The same is obviously true on $N$. Therefore $(M, \mathcal{F})$ is holomorphically plain.

1.5. An example. The results described so far might lead one to expect that holomorphically foliated spaces are holomorphically plain under very general conditions and that one should be able to prove it using only qualitative properties of leafwise holomorphic functions. The next theorem shows, however, that the situation cannot be so simple.

Theorem 1.16. There exists a compact real analytic foliation $(M, \mathcal{F})$, which is a foliated bundle over a compact Riemann surface, and a real analytic leafwise holomorphic function $f: M \rightarrow \mathbb{C}$ that is not leafwise constant.

To construct an example of $(M, \mathcal{F})$ and $f$ as in Theorem 1.16 we first introduce some notation. Let $\mathbb{D}$ denote, as before, the unit open disk in $\mathbb{C}$. Points in projective space $\mathbb{R} P^{4}$ will be written $\left[z_{1}, z_{2}, t\right]$, where $z_{i} \in \mathbb{C}, t \in \mathbb{R}$ and $\left(z_{1}, z_{2}, t\right)$ is nonzero. Define a (real analytic) action of $S U(1,1)$ on $\mathbb{R} P^{4}$ as follows. Elements of $S U(1,1)$ are matrices of the form $\left(\begin{array}{cc}\alpha & \beta \\ \bar{\beta} & \bar{\alpha}\end{array}\right)$ for which $|\alpha|^{2}-|\beta|^{2}=1$. The action of $S U(1,1)$ on $\mathbb{R} P^{4}$ defined by

$$
\left(\begin{array}{cc}
\alpha & \beta \\
\bar{\beta} & \bar{\alpha}
\end{array}\right) \cdot\left[z_{1}, z_{2}, t\right]:=\left[\alpha z_{1}+\beta \bar{z}_{2}, \alpha z_{2}+\beta \bar{z}_{1}, t\right]
$$

leaves invariant the submanifold $\mathcal{C}:=\left\{\left[z_{1}, z_{2}, t\right] \in \mathbb{R} P^{4}:\left|z_{1}\right|^{2}-\left|z_{2}\right|^{2}=t^{2}\right\}$.

We define on $\mathbb{D} \times \mathcal{C}$ the function

$$
f(z,[\alpha, \beta, t]):=\frac{\bar{\alpha} z-\beta}{-\bar{\beta} z+\alpha} .
$$

An elementary calculation shows that $f(g z, g[\alpha, \beta, t])=f(z,[\alpha, \beta, t])$ for every $g \in$ $S U(1,1)$. Therefore, if $\Gamma$ is a uniform lattice in $S U(1,1)$, then $f$ yields a function on the foliated bundle $M=(\mathbb{D} \times \mathcal{C}) / \Gamma$ that is real analytic, leafwise holomorphic and the restriction of $f$ to any leaf for which $t \neq 0$ is not constant.

1.6. A universal non-plain foliated space. We describe now a kind of "universal space" from which such examples can be constructed. This will be done in the setting of foliated bundles whose base are compact Riemann surfaces, but it should be apparent that the same ideas apply more broadly. Let $X_{0}:=\operatorname{Hol}(\mathbb{D}, \overline{\mathbb{D}})$ be the space of holomorphic functions defined on $\mathbb{D}$ such that $\sup \{|f(z)|: z \in \mathbb{D}\} \leq 1$. Then $X_{0}$, with the topology of uniform convergence on compact sets, is a compact metrizable space on which $P S U(1,1)$ acts via the continuous action $(g, f) \mapsto f \circ g^{-1}$, where $g$, on the right-hand side, is regarded as an automorphism (a Möbius transformation) of the Poincaré disc.

Now let $\rho: \Gamma \rightarrow P S U(1,1)$ be a homomorphism from the fundamental group of a compact Riemann surface $S=\mathbb{D} / \Gamma$ into the Möbius group, and construct the 
foliated bundle $\left(\tilde{S} \times X_{0}\right) / \Gamma$ over $S$. We will denote the resulting foliated space by $\left(M_{0}, \mathcal{F}_{0}\right)$. By a morphism $f:(M, \mathcal{F}) \rightarrow\left(M_{0}, \mathcal{F}_{0}\right)$ we will mean a (continuous) $f: M \rightarrow M_{0}$ that maps leaves to leaves holomorphically such that $\pi_{M_{0}} \circ f=\pi_{M}$, where $\pi_{M}$ (resp., $\pi_{M_{0}}$ ) is the natural projection from $M$ to $S$ (resp., from $M_{0}$ to $S)$.

A leafwise nonconstant, leafwise holomorphic function can now be produced on $\left(M_{0}, \mathcal{F}_{0}\right)$ by the following essentially tautological procedure. First define $\bar{\phi}$ : $\mathbb{D} \times X_{0} \rightarrow \mathbb{C}$ by $\bar{\phi}(z, f):=f(z)$. Note that $\bar{\phi}\left(\gamma(z), f \circ \gamma^{-1}\right)=\bar{\phi}(z, f)$ for each $\gamma \in \Gamma$. There is as a result a well-defined function $\phi: M_{0} \rightarrow \mathbb{C}$ such that $\phi \circ \pi=\bar{\phi}$, where $\pi$ is the natural projection from $\mathbb{D} \times X_{0} \rightarrow M_{0}$. The function $\phi: M_{0} \rightarrow \mathbb{C}$ is a continuous, leafwise holomorphic function.

The following remark is an immediate consequence of these definitions. In the proposition, equivariance of a map $\hat{\psi}: V \rightarrow X_{0}$ means that, for each $v \in V$ and $\gamma \in \Gamma, \hat{\psi}(\gamma(v))=\gamma \circ \hat{\psi}(v)$.

Proposition 1.17. Let $(M, \mathcal{F})$ be a foliated bundle over $\mathbb{D} / \Gamma$ with fiber $V$. Then there is a one-to-one correspondence between (continuous) leafwise holomorphic functions $\psi: M \rightarrow \mathbb{C}$ having absolute value bounded above by 1 and $\Gamma$-equivariant (continuous) $\hat{\psi}: V \rightarrow X_{0}$. Furthermore, if $\Psi: M \rightarrow M_{0}$ is the morphism of holomorphically foliated spaces induced from $\hat{\psi}$, then $\psi=\phi \circ \Psi$, and $\Psi$ is the unique morphism from $M$ to $M_{0}$ that satisfies this last equality.

The proposition indicates how to go about looking for examples of foliated manifolds that are not holomorphically plain: one tries to find a $\Gamma$-invariant manifold $V$ embedded in $X$. Specifically, one can try to obtain a manifold $V \subset X$ as the closure of a $P S U(1,1)$-orbit. In fact, the example given just after Theorem 1.16 is closely related to what one gets by taking $V$ to be the closure of the $P S U(1,1)$-orbit of the function $\varphi(z)=z$ in $\operatorname{Hol}(\mathbb{D}, \overline{\mathbb{D}})$. This closure is the compactification of a 3-manifold (an open solid torus) by adding a circle at infinity (so as to form a 3 -sphere), while the submanifold $\mathcal{C} \subset \mathbb{R} P^{4}$ of that example corresponds to an analytic "doubling" of this 3-manifold.

The remark just made suggests that a precise characterization of holomorphically plain foliated bundles over a compact Riemann surface will require an investigation of the dynamics of the action of $P S U(1,1)$ on $\operatorname{Hol}(\mathbb{D}, \overline{\mathbb{D}})$. Such a characterization should tell, in particular, how common holomorphically plain foliations are, at least in this special setting.

\section{REFERENCES}

[1] N. A'Campo and M. Burger. Réseaux arithmétiques et commensurateur d'après G. A. Margulis, Invent. Math. 116 (1994) 1-25. MR 96a:22019

[2] A. Candel. The Harmonic measures of Lucy Garnett, preprint, 2000.

[3] A. Candel and L. Conlon. Foliations I, Graduate Studies in Mathematics, Volume 23, AMS, 2000. MR 2002f: 57058

[4] D. Cerveau, E. Ghys, N. Sibony, J.C. Yoccoz. Dynamique et Géométrie Complexes, Panoramas et Synthèses, Société Mathématique de France, 1999. MR 2001a:37002

[5] A. Connes. A survey of foliations and operator algebras, Proc. Symp. Pure Math., Amer. Math. Soc. (1982) 521-628. MR 84m:58140

[6] L. Garnett. Foliations, the ergodic theorem and Brownian motion, J. Funct. Anal. 51 (1983), 285-311. MR 84j:58099

[7] E. Ghys and P. de la Harpe. Sur les Groups Hyperboliques d'après Mikhael Gromov, Progress in Mathematics 83, Birkhäuser, Basel, 1990. MR 92f:53050 
[8] P. Griffiths and J. Harris. Principles of Algebraic Geometry, John Wiley \& Sons, 1994. MR 95d:14001

[9] A. Haefliger. Variétés feuilletées, Ann. Scuola Norm. Sup. Pisa 16 (1962) 367-397. MR 32:6487

[10] G. Hector and U. Hirsch. Introduction to the Geometric Theory of Foliations, Aspects of Mathematics, 1983, Vieweg. MR 85f:57016

[11] K. Kodaira. Complex Manifolds and Deformations of Complex Structures, Springer, 1986. MR 87d:32040

[12] G. A. Margulis. Discrete Subgroups of Semisimple Lie Groups, Springer, 1989.

[13] Pierre Molino. Riemannian Foliations, Birkhäuser, 1987. MR 89b:53054

[14] A. S. Rapinchuk, V. V. Benyash-Krivetz, V. I. Chernousov. Representation varieties of the fundamental groups of compact orientable surfaces, Israel J. Math. 93 (1996) 29-71. MR 98a:57002

Department of Mathematics-1146, Washington University, St. Louis, Missouri 63130

UMpa - École Normale Supérieure de Lyon, 69364 Lyon Cedex 07, France 